\title{
Nanosecond laser-induced periodic surface structures on wide band-gap semiconductors
}

\author{
Mikel Sanz ${ }^{1 *}$, Esther Rebollar $^{1}$, Rashid A. Ganeev $^{2}$, Marta Castillejo $^{1}$ \\ ${ }^{1}$ Instituto de Química Física Rocasolano, CSIC, Serrano 119, 28006 Madrid, Spain \\ ${ }^{2}$ Voronezh State University, Voronezh 394006, Russia \\ *Email: mikel.sanz@iqfr.csic.es
}

Tel.: +34915619400961023

Fax: +34915642461

\begin{abstract}
In this work we report on fabrication of laser-induced periodic surface structures (LIPSS) on different semiconductors with bandgap energies in the range of 1.3-3.3.eV and melting temperatures from 1100 to $2700{ }^{\circ} \mathrm{C}$. In particular, InP, GaAs, GaP and SiC were irradiated in air with nanosecond pulses using a linearly polarized laser beam at $266 \mathrm{~nm}$ (6 ns pulse width). The nanostructures, inspected by atomic force microscopy, are produced upon multiple pulse irradiation at fluences near the ablation threshold. LIPSS are perpendicular to the laser polarization direction and their period is of the order of the irradiation wavelength. It was observed that the accumulative effect of both fluence and number of pulses needed for LIPSS formation increased with the material bandgap energy. These results, together with estimations of surface temperature increase, are discussed with reference to the semiconductor electrical, optical and thermal properties.
\end{abstract}

\section{Keywords}


Laser-induced periodic surface structures; Nanosecond pulsed laser irradiation; Semiconductors

\section{Introduction}

Since the early observation of laser-induced periodic surface structures (LIPSS), also termed as ripples, on semiconductors [1] this kind of nanostructures have been imprinted on almost all kinds of materials and have been extensively investigated using low power cw and pulsed laser sources of nanosecond (ns) and femtosecond (fs) duration [2-8]. In general, the ripples have a period $\Lambda$ dependent on laser wavelength $\lambda$, on the angle of incidence of the radiation $\Theta_{i}$ and on index of refraction $n$ and can be described by the relation $\Lambda=\lambda /\left(n-\sin \Theta_{\mathrm{i}}\right)$ [2]. After exposure of a smooth solid to a linearly polarized radiation at normal incidence, often the lateral period of the fabricated LIPSS is very close to the wavelength of the incident radiation. It has been proposed that this type of ripples arises from optical interference effects due to the superposition of the incident radiation with a surface electromagnetic wave which is created at the material-medium interface during irradiation together with a feedback mechanism [3-8]. Recently, LIPSS resulting from fs laser irradiation of solids have received considerable attention in attempts to determine their formation mechanism [9-19]. Irradiation of surfaces at normal incidence usually leads to the formation of low spatial frequency LIPSS (LSFL) with period comparable to the laser wavelength. This type of structures is explained in reference to the previously mentioned interference mechanism. In the case of metals, semiconductors and dielectrics, the formation of ripple structures with subwavelength periods has also been observed. These high spatial frequency LIPSS (HSFL) have been obtained using fs laser pulses of different duration, wavelength, fluence and number of pulses [20-25]. Several mechanisms have been proposed as the origin of HSFL, such as interference effects along with transient changes in the optical 
properties during laser irradiation [26], second harmonic generation [24,27], excitation of surface plasmon polaritons [28], resolidification [29], self organization [11-13] and Coulomb explosion [30].

Wide band gap (WBG) semiconductors have expanded the scope of applications beyond those of silicon. The developing list of such materials for use in device production is remarkable and continues to provide new design possibilities. The inherent properties of WBG make them ideal candidates for high-power, high-temperature electronic devices, power amplifiers, switches, and short wavelength light sources. Therefore, modification of structure and properties of WBGs at the nanometer scale attracts great interest [31]. In this work, LIPSS were imprinted on the surface of WBG semiconductors wafers of Indium Phosphide (InP), Gallium Arsenide (GaAs), Gallium Phosphide (GaP) and Silicon Carbide (SiC) by irradiating in air with linearly polarized, $266 \mathrm{~nm}, 6 \mathrm{~ns}$ laser pulses. The period and amplitude of the LIPSS were characterized by atomic force microscopy (AFM) as a function of the laser fluence and number of pulses. We have observed that as the bandgap of the semiconductor material increases, higher fluence or number of pulses are needed for LIPSS formation whereas the amplitude of the ripples is related with the optical and thermal penetration depth. Estimations of surface temperature increase are discussed with reference to the WBG semiconductor electrical, optical and thermal properties.

\section{Experimental setup}

For studying ripple formation, multiple pulse laser irradiation of undoped semiconductor wafers of $\mathrm{InP}, \mathrm{GaAs}, \mathrm{GaP}$ and $\mathrm{SiC}$ was carried out in ambient air at normal incidence. The electrical, optical and thermal properties of those materials $[32,33]$ are summarized in Table 1 . For irradiating the samples we used the linearly 
polarized fourth harmonic output of a Q-switched Nd:YAG laser (Quantel Brilliant B, $266 \mathrm{~nm}, \tau=6 \mathrm{~ns}$ FWHM, repetition rate $10 \mathrm{~Hz}$, Gaussian beam profile). This irradiation wavelength corresponds to an energy of $4.67 \mathrm{eV}$, well above the bandgap energies of these semiconducting materials (Table 1). The central (4 mm diameter) most uniform part of the beam spot was selected for irradiation by using a diaphragm. The laser beam was focused on the substrate surface with a spherical lens of $15 \mathrm{~cm}$ focal length. The irradiation fluence was below the ablation threshold $\left(F_{t h}\right)$ for a single pulse of each material. The fluence was determined as the ratio of the laser pulse energy, measured in front of the sample with a joulemeter (Gentec-E, QE25SP-H-MB-D0), and the area of the irradiated spot, determined by the print left on an unplasticized polyvinyl chloride sheet. Ablation thresholds of samples were determined by measuring the minimum single pulse energy necessary to yield a surface change as detected by optical microscopy (Leica, S8APO) with a 160x microscope objective and equipped with a CCD camera. The obtained values are $190 \pm 10,380 \pm 22,470 \pm 25$ and $950 \pm 40$ $\mathrm{mJ} / \mathrm{cm}^{2}$, for InP, GaAs, GaP and $\mathrm{SiC}$, respectively. The morphology of the laser treated semiconductor surfaces was characterized using AFM in tapping mode (Multimode 8, Bruker) and the images were analysed with the software Nanoscope Analysis 1.40. The AFM measurements were performed in 5 different positions of each sample to check the uniformity of the fabricated nanostructures. The pristine substrates, of around $300 \mu \mathrm{m}$ thick, present a flat surface, with mean roughness $\left(R_{a}\right)$ values $<1 \mathrm{~nm} . R_{a}$ values are listed in Table 1 and indicate the arithmetic average of the deviations in height from the center plane of the sample. Each $R_{a}$ value corresponds to the average of three independent measurements in different locations of the substrate surface.

\section{Results}


Irradiation of the semiconductor wafers was performed at different fluences and number of pulses in order to find the conditions for obtaining the most uniform ripples in terms of period and amplitude. The minimum fluence $\left(F_{m}\right)$ needed for LIPPS fabrication is displayed in Table 2 together with the experimental conditions for the optimum LIPSS fabrication for each semiconductor wafer. Figure 1 shows AFM height images and corresponding cross-section of the LIPSS obtained in InP, GaAs and GaP.

Figure 1a displays the ripples fabricated in InP with 200 pulses of $125 \mathrm{~mJ} / \mathrm{cm}^{2}$. Ripples are perpendicular to the laser polarization direction and the measured period was estimated in $248 \mathrm{~nm}$ with amplitude of $5 \mathrm{~nm}$. The structures were observed at fluences of $100-150 \mathrm{~mJ} / \mathrm{cm}^{2}$ with $100-300$ pulses.

In the case of GaAs, the optimum conditions for LIPSS formation of $\operatorname{InP}\left(125 \mathrm{~mJ} / \mathrm{cm}^{2}\right.$, 200 pulses) resulted in isolated rounded nanostructures (Figure 2a). When increasing the number of pulses, the ripples started to form and align (Figure 2b) whereas the increase of fluence finally results in LIPSS formation (Figure $1 \mathrm{~b}$ and 2c). Further fluence increase induced the destruction of the nanostructures (Figure 2d). The best ripples were obtained with 200 pulses at $150 \mathrm{~mJ} / \mathrm{cm}^{2}$. Under these conditions the estimated period was $253 \mathrm{~nm}$ and the amplitude $9 \mathrm{~nm}$.

For GaP, a number of pulses higher than that used for GaAs are necessary to fabricate uniform LIPSS. The best ripples, with a period of $263 \mathrm{~nm}$ and amplitude of $15 \mathrm{~nm}$, were obtained at $125 \mathrm{~mJ} / \mathrm{cm}^{2}$ and 300 pulses (Figure 1c). Increasing the fluence and/or the number of pulses caused the disappearance of the uniform ripples.

In the case of $\mathrm{SiC}$, no LIPSS were obtained for fluences as large as $300 \mathrm{~mJ} / \mathrm{cm}^{2}$ and for a large number of pulses (up to 600).

\section{Discussion}


As mentioned, the mechanism of LIPSS formation can be explained as the result of the optical interference effects due to the superposition of the incident radiation with a surface electromagnetic wave, which is created and scattered along the irradiated surface $[2,5,34]$. This results in a modulated distribution of energy on the surface which consequently induces a similarly modulated heating. The thermal penetration depth of the irradiated zone can be calculated by $d_{t h}=(D \tau)^{1 / 2}$ with $D=\kappa / \rho c$ being the thermal diffusivity, $\kappa$ the thermal conductivity, $\rho$ the density, $c$ the specific heat of the material (Table 1) and $\tau$ the pulse duration (6 ns). For the materials under study $d_{t h}$ is below 1 $\mu \mathrm{m}$, thus much smaller than the diameter of the irradiated area. Therefore, for estimating the temperature increase of the irradiated substrate, one can assume for simplicity that the temperature distribution is associated with depth $x$ and time $t$. It is also assumed that, during the irradiation time interval, the material parameters and the irradiation intensity are constant. Under these approximations, the one-dimensional heat conduction equation can be used to estimate the temperature increase as function of $x$ and $t[35,36]$ :

$$
\frac{\partial^{2} T(x, t)}{\partial x^{2}}-a^{2} \frac{\partial T(x, t)}{\partial T}=-\frac{\alpha}{\kappa} P(t) \exp (-\alpha x) F_{0}
$$

with $a^{2}=\rho c / \kappa . \quad P(t)=\left(2 t / \tau^{2}\right) \exp \left[-(t / \tau)^{2}\right]$ is the function describing the temporal shape of laser pulse [36], approximated by a modified Gaussian beam, where the $t$ factor ensures that intensity vanishes at $t=0 . F_{o}$ is the used laser fluence. The time dependence of the temperature can be obtained by solving the previous equation as described in ref. 36 . Figure 3 shows for the materials studied herein the temporal evolution of the estimated surface temperature upon irradiation with a single laser pulse of $266 \mathrm{~nm}$ at $125 \mathrm{~mJ} / \mathrm{cm}^{2}$. 
The maxima of the curves correspond to the maxima temperature attained in the surface of the different samples.

The optimum conditions for LIPSS formation can be related with the surface temperature reached upon irradiation and with the semiconductor melting point (see Table 1). For InP and GaAs, the temperature attained at the surface is slightly below the corresponding melting point (Table 1). In the case of GaP, the temperature of the irradiated surface is clearly below the melting point. For $\mathrm{SiC}$, and due to the high values of specific heat $c$ and thermal conductivity $k$ of this material, higher fluences than those explored in this work are expected to be required to melt the outer sample layer.

These results indicate that in order to obtain LIPSS, a minimum fluence is necessary to assure that the surface temperature is high enough for allowing melting and material rearrangement. On the other hand, material evaporation produces emission of atoms from the semiconductor surface [34]. Evaporation is the origin of microdefects that raise the surface roughness and enhance the absorption of the produced microsites. This in turn increases the surface inhomogeneities which facilitate the feedback mechanism necessary for the ripple formation.

It is important to notice that temperature estimations do not take into account neither modification of the thermal properties of the material nor incubation effects that can be induced as a consequence of repetitive irradiation. In order to assess the influence of multiple pulse irradiation, we calculated the thermal diffusion time, $t_{d}=1 / D \alpha^{2}$. This parameter is of the order of $100 \mu$ s for the studied materials. These thermal diffusion times are much smaller than the time interval between two consecutive pulses (100 ms). This implies that purely cumulative thermal heating can be excluded as the origin of ripples. 
The relative increase of fluence and number of pulses needed for imprinting optimum LIPSS on the semiconductors are observed to rise as the energy bandgap increases. This is related to the fact that higher melting temperatures correspond to wider bandgaps (Table 1) and thus, higher temperatures should be reached upon irradiation in order to allow melting and rearrangement of material.

Measured ripple periods are of the order of the irradiation wavelength for all analyzed semiconductors, whereas a moderate increase in amplitude is observed as the optical and thermal penetration depths of the material increase. The optical absorption depth for a single pulse, calculated as the inverse of the linear optical absorption coefficient (Table 1), is larger for GaP than for InP and GaAs. The thermal penetration depth $d_{t h}$ is also higher for $\mathrm{GaP}(610 \mathrm{~nm})$ than for $\mathrm{InP}$ and $\mathrm{GaAs}$ (around $450 \mathrm{~nm}$ ) for the given irradiation conditions. The larger optical and thermal penetration depths induce a deeper effect close to the surface in $\mathrm{GaP}$ as compared to $\mathrm{InP}$ and $\mathrm{GaAs}$, in good agreement with the observed ripple amplitude values: the ripple amplitude for $\mathrm{GaP}$ is $15 \mathrm{~nm}$, whereas 5 $\mathrm{nm}$ amplitude ripples are obtained in the case of $\mathrm{InP}$.

\section{Conclusions}

Laser-induced periodic surface structures perpendicular to the laser polarization direction and with period of the order of the irradiation wavelength were obtained on the surface of wide bandgap semiconductor wafers of InP, GaAs, GaP and SiC upon repetitive irradiation at $266 \mathrm{~nm}$ with pulses of $6 \mathrm{~ns}$. The ripples were fabricated at fluences below the ablation threshold for a single pulse irradiation and are produced mainly by optical interference effects due to the superposition of the incident radiation with a surface electromagnetic wave created on the wafer surface. Calculation of the temperature increase induced by laser irradiation indicates that a minimum fluence is 
necessary to assure that the surface temperature is high enough for allowing melting and material rearrangement. The increased surface inhomogeneity caused by microdefects created by laser irradiation facilitates the feedback mechanism generating the ripple formation. The higher fluence and number of pulses needed for LIPSS formation as the bandgap increases is related to the higher melting temperatures corresponding to larger bandgaps. It was observed that the amplitude of the ripples increases with optical and thermal penetration depths.

\section{Acknowledgements}

We acknowledge funding from Ministry of Science and Innovation of Spain (MICINN) under Project CTQ2010-15680. E.R and M.S. gratefully thank Juan de la Cierva Programme (MICINN), CONSOLIDER CSD2007-00058, and Geomateriales (CAM, S2009/Mat-1629) for contracts. We are grateful to Prof. T. Ezquerra (IEM, CSIC) for the use of the AFM system.

\section{References}

[1] M. Birnbaum, J. Appl. Phys. 36 (1965) 3688.

[2] D. Bäuerle, Laser Processing and Chemistry; fourth ed., Springer-Verlag: Berlin Heidelberg, 2011.

[3] D.C. Emmony, R.P. Howson, L.J. Willis, Appl. Phys. Lett. 23 (1973) 598.

[4] Z. Guosheng, P.M. Fauchet, A.E. Siegman, Phys. Rev. B 26 (1982) 5366.

[5] J.E. Sipe, J.F. Young, J.S. Preston, H.M. van Driel, Phys. Rev. B 27 (1983) 1141.

[6] J.F. Young, J.S. Preston, H.M. van Driel, J.E. Sipe, Phys. Rev. B 27 (1983)1155.

[7] S.E. Clark, D.C. Emmony, Phys. Rev. B 40 (1989) 2031.

[8] B. Kumar, R.K. Soni, J. Phys. D: Appl. Phys. 41 (2008) 155303. 
[9] A.M. Ozkan, A.P. Malshe, T.A. Railkar, W.D. Brown, M.D. Shirk, P.A. Molian, Appl. Phys. Lett. 75 (1999) 3716.

[10] J. Bonse, J.M. Wrobel, K.-W. Brzezinka, N. Esser, W. Kautek, Appl. Surf. Sci. 202 (2002) 272.

[11] J. Reif, F. Costache, M. Henyk, S.V. Pandelov, Appl. Surf. Sci. 197-198 (2002) 891.

[12] F. Costache, M. Henyk, J. Reif, Appl. Surf. Sci. 208-209 (2003) 486.

[13] O. Varlamova, F. Costache, J. Reif, M. Bestehorn, Appl. Surf. Sci. 252 (2006) 4702.

[14] M. Groenendijk, J. Meijer, J. Laser Appl. 18 (2006) 227.

[15] T. Tomita, Y. Fukumori, K. Kinoshita, S. Matsuo, S. Hashimoto, Appl. Phys. Lett. $92(2008) 013104$.

[16] J. Bonse, A. Rosenfeld, J. Krüger, J. Appl. Phys. 106 (2009) 104910.

[17] J. Reif, O. Varlamova, F. Costache, Appl. Phys. A 92 (2008) 1019.

[18] M. Huang, F. Zhao, Y. Cheng, N. Xu, Z. Xu, ACS Nano 3 (2009) 4062.

[19] R.A. Ganeev, M. Babu, T. Ozaki, H. Kuroda, J. Opt. Soc. Am. B 27 (2010) 1077.

[20] E.M. Hsu, T.H.R. Crawford, H.F. Tiedje, H.K. Haugen, Appl. Phys. Lett. 91 (2007) 111102.

[21] U. Chakravarty, R.A. Ganeev, P.A. Naik, J.A. Chakera, M. Babu, P.D. Gupta, J. Appl. Phys. 109 (2011) 084347.

[22] N. Yasumaru, K. Miyazaki, J. Kiuchi, Appl. Phys. A 81 (2005) 933.

[23] M. Shinoda, R.R. Gattass, E. Mazur, J. Appl. Phys. 105 (2009) 053102.

[24] A. Borowiec, H.K. Haugen, Appl. Phys. Lett. 82 (2003) 4462.

[25] J. Reif, O. Varlamova, M. Ratzke, M. Schade, H.S. Leipner, T. Arguinov, Appl. Phys. A 101 (2010) 361. 
[26] Q. Wu, Y. Ma, R. Fang, Y. Liao, Q. Yu, X. Chen, K. Wang, Appl. Phys. Lett. 82 (2003) 1703.

[27] J. Bonse, M. Munz, H. Sturm, J. Appl. Phys. 97 (2005) 013538.

[28] G. Miyaji, K. Miyazaki, Opt. Express 16 (2008) 16265.

[29] J. Bonse, K.-W. Brzezinka, A.J. Meixner, Appl. Surf. Sci. 221 (2004) 215.

[30] Y. Dong, P. Molian, Appl. Phys. Lett. 84 (2004) 10.

[31] D. Bimberg, Semiconductor Nanostructures, Springer-Verlag: Berlin Heidelberg, 2008.

[32] E.D. Palik, Handbook of Optical Constants of Solids; Academic Press, New York, 1997.

[33] S.O. Kasap, P. Capper, Handbook of Electronic and Photonic Materials; SpringerVerlag Berlin Heidelberg 2006.

[34] H. Qi, Q. Wang, X. Zhang, Z. Liu, S. Zhang, J. Chang, W. Xia. G. Jin, Opt. Lasers Eng. 49 (2011) 285.

[35] M. Csete, Zs. Bor, App. Surf. Sci. 133 (1998) 5.

[36] E. Rebollar, S. Perez, J.J. Hernandez, I. Martin-Fabiani, D.R. Rueda, T.A. Ezquerra, M. Castillejo, Langmuir 27 (2011) 2996. 
Table 1. Initial roughness ${ }^{\mathrm{a}}\left(R_{a}\right)$, energy bandgap [33], melting temperature [33] $\left(T_{m}\right)$, specific heat [33] $(c)$, thermal conductivity [33] $(k)$, density [33] $(\rho)$ and linear absorption coefficient at $266 \mathrm{~nm}[32](\alpha)$ of semiconductor wafers.

\begin{tabular}{lccccccc}
\hline Material & $\begin{array}{c}R_{a} \\
(\mathrm{~nm})\end{array}$ & $\begin{array}{c}\text { Bandgap } \\
(\mathrm{eV})\end{array}$ & $\begin{array}{c}T_{m} \\
\left({ }^{\circ} \mathrm{C}\right)\end{array}$ & $\begin{array}{c}c \\
(\mathrm{~J} / \mathrm{Kg} \mathrm{K})\end{array}$ & $\begin{array}{c}k \\
(\mathrm{~W} / \mathrm{m} \mathrm{K})\end{array}$ & $\begin{array}{c}\rho \\
\left(\mathrm{Kg} / \mathrm{m}^{3}\right)\end{array}$ & $\begin{array}{c}\alpha \\
\left(\mathrm{cm}^{-1}\right)\end{array}$ \\
\hline $\mathrm{InP}$ & 0.30 & 1.35 & 1060 & 310 & 68 & 4810 & $1.47 \cdot 10^{6}$ \\
$\mathrm{GaAs}$ & 0.28 & 1.42 & 1240 & 330 & 55 & 5316 & $1.72 \cdot 10^{6}$ \\
$\mathrm{GaP}$ & 0.62 & 2.30 & 1457 & 430 & 110 & 4138 & $1.21 \cdot 10^{6}$ \\
$\mathrm{SiC}$ & 0.65 & 3.37 & 2730 & 690 & 370 & 3210 & $2.44 \cdot 10^{6}$ \\
\hline
\end{tabular}

${ }^{\mathrm{a}}$ Estimated by AFM. 
Table 2. Minimal fluence $\left(F_{m}\right)$ required for LIPSS fabrication and experimental conditions (fluence and number of pulses) and ripple properties (period and amplitude) for optimum LIPSS of semiconductor wafers.

\begin{tabular}{lccccc}
\hline Material & $\begin{array}{c}F_{m} \\
\left(\mathrm{~mJ} / \mathrm{cm}^{2}\right)\end{array}$ & $\begin{array}{c}\text { Fluence } \\
\left(\mathrm{mJ} / \mathrm{cm}^{2}\right)\end{array}$ & $\begin{array}{c}\text { Number } \\
\text { of pulses }\end{array}$ & $\begin{array}{c}\text { Ripple } \\
\text { period }(\mathrm{nm})\end{array}$ & $\begin{array}{c}\text { Ripple } \\
\text { amplitude }(\mathrm{nm})\end{array}$ \\
\hline $\mathrm{InP}$ & 100 & 125 & 200 & $248 \pm 6$ & $5 \pm 1$ \\
$\mathrm{GaAs}$ & 135 & 150 & 200 & $253 \pm 7$ & $9 \pm 2$ \\
$\mathrm{GaP}$ & 125 & 125 & 300 & $263 \pm 10$ & $15 \pm 5$ \\
$\mathrm{SiC}$ & $>300$ & - & - & & No LIPSS Observed \\
\hline
\end{tabular}




\section{Figure captions}

Figure 1. AFM height images ( $2 \times 2 \mu m^{2}$ size) (left) and corresponding cross-sections (right) of LIPSS fabricated at $266 \mathrm{~nm}$ in (a) InP with 200 pulses at a fluence of 125 $\mathrm{mJ} / \mathrm{cm}^{2}$ (b) GaAs with 200 pulses at $150 \mathrm{~mJ} / \mathrm{cm}^{2}$, and (c) GaP with 300 pulses at 125 $\mathrm{mJ} / \mathrm{cm}^{2}$. The ripples are perpendicular to the laser polarization direction.

Figure 2. AFM height images ( $3 \times 3 \mu \mathrm{m}^{2}$ size) of LIPSS fabricated at $266 \mathrm{~nm}$ in GaAs at the indicated conditions. The ripples are perpendicular to the laser polarization direction.

Figure 3. Time dependence of the temperature reached on the semiconductor surface

under irradiation with a single pulse of $266 \mathrm{~nm}$ at $125 \mathrm{~mJ} / \mathrm{cm}^{2}$, for the different materials, as indicated. 
Figure 1
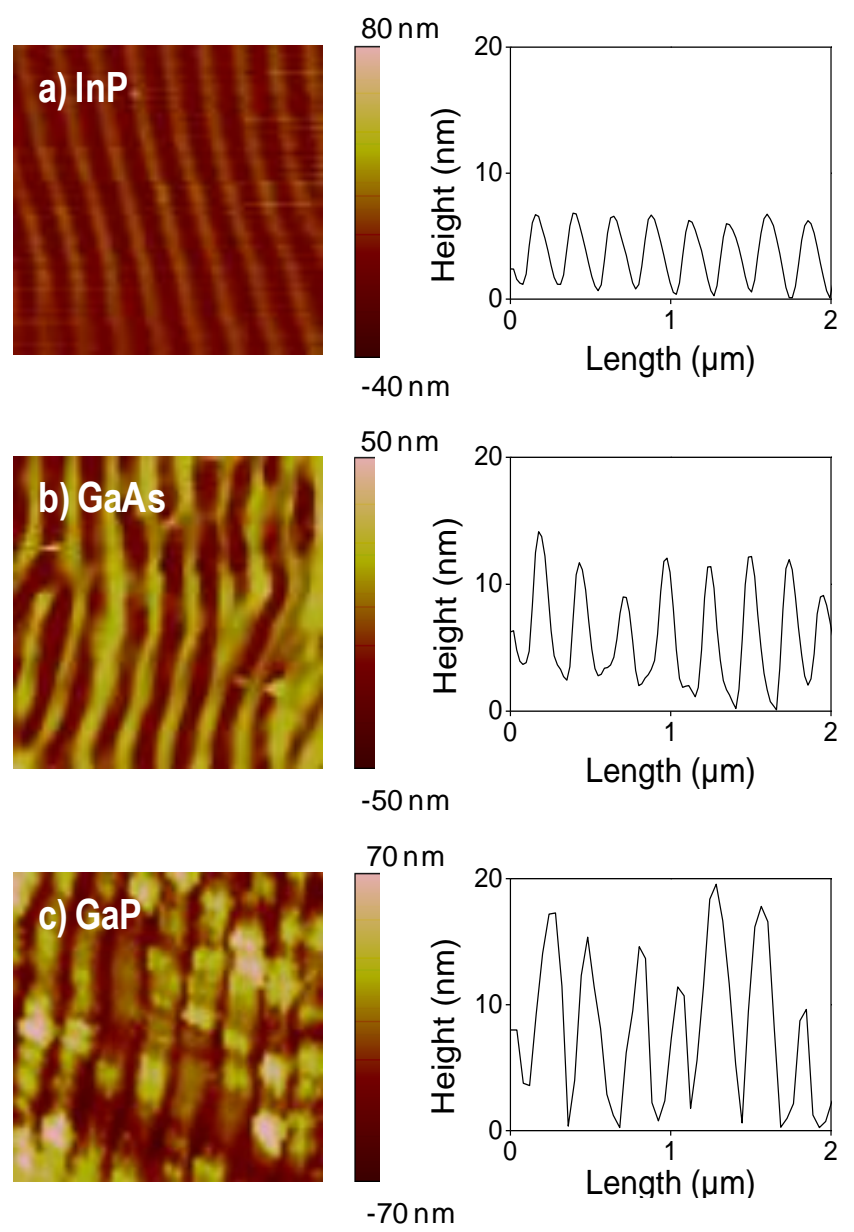
Figure 2

$80 \mathrm{~nm}$
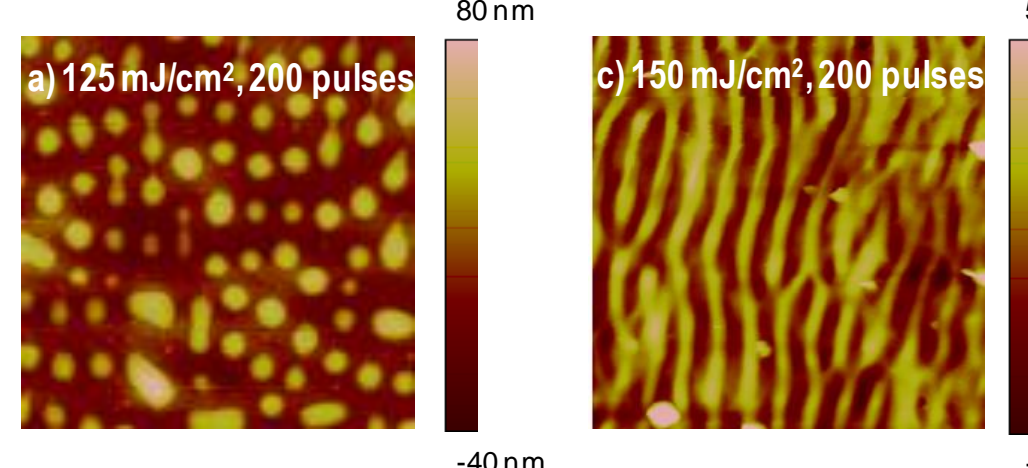

$-40 \mathrm{~nm}$

$68 \mathrm{~nm}$

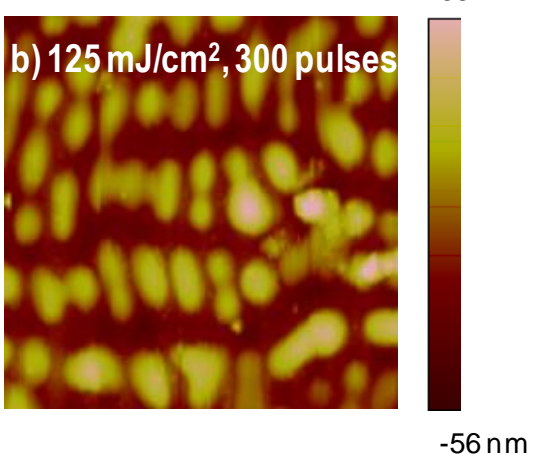

$50 \mathrm{~nm}$

$-50 \mathrm{~nm}$

$170 \mathrm{~nm}$

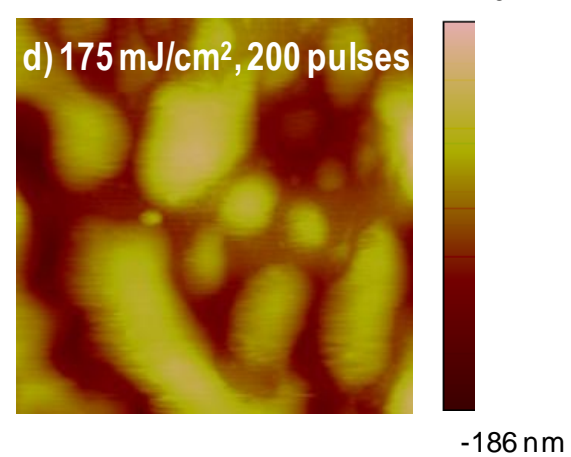


Figure 3

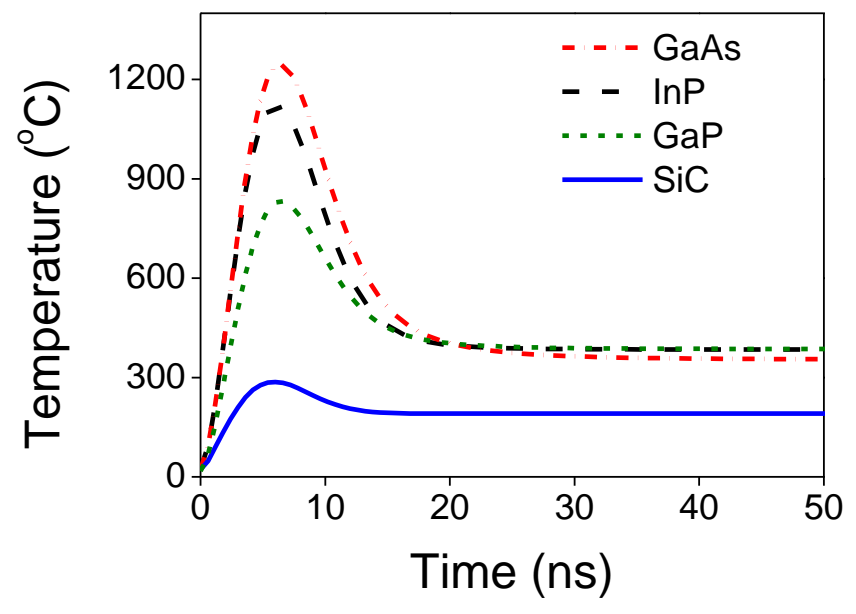

